\title{
Division of Reproductive Science Research
}

The Reproductive Science Research Division in the Department of Obstetrics and Gynecology was established in 2009 and consists of four full time research faculty members and eight faculty members with joint appointment with other divisions in the department.

Members in the Research Division conducted research in diverse areas of basic reproductive research:

Dr. Bradley: Female urinary incontinence and other pelvic floor disorders.

Dr. Dai: Novel prognostic indicator integrating genome sequencing data and tumor evolution history for prediction of endometrial cancer outcome.

Dr. Devor: The role of microRNAs and other non-coding RNAs, specifically $\mathrm{H} 19$ and miR-675, in differentiating among endometrial cancers. Developing miR-675 as a potential biomarker of more aggressive tumors.

Dr. Goodheart: The role of Lef- 1 in endometrial gland formation and cancer. Angiogenic markers in ovarian cancer.

Division Activities

Department of Obstetrics and Gynecology The University of lowa, lowa City, IA
Dr. Hunter: Non-dietary treatment of mPKU; the vaccine development against GBS; the mechanism and potential treatment of preeclampsia.

Dr. Leslie: The molecular biology of estrogen and progesterone receptors in the treatment of endometrial cancer, and identify molecular targets linked to response in clinical trials.

Dr. Meng: New prognosis biomarkers and potential therapeutic targets in gynecology; restore progesterone receptor gene expression to improve the efficacy of hormone therapy in endometrial cancer; synthetic lethal studies in gynecology.

Dr. D. Santillan: Development of a GBS vaccine, Understanding the role of Placental Hematopoietic Stem Cells, Developing a MaternalFetal Tissue Bank, Immune Modulators of Preeclampsia.

Dr. M. Santillan: Investigating the role of immunology in the pathogenesis of preeclampsia, determining molecular mechanisms of vascular function changes in mice and humans throughout pregnancy, development of a 
prospectively collected Maternal

Fetal Tissue Bank and Clinical

Database.

Dr. Sparks: Gamete biology; in vitro fertilization; embryo culture; and gamete/embryo cryopreservation

Dr. Stockdale: Vulvar and lower genital tract disease.

Dr. Yang: Development of mouse iPS cells; the mechanism and potential treatment of saltsensitive hypertension; IGF-1 signaling regulation by Nedd4 during normal and malignant growth.

Baoli Yang, MD, PhD

Division Director, Reproductive Science Research 